\title{
A qualidade da sistematização da assistênca em enfemagem em um hospital público de Teresina
}

\author{
The quality of nursing care systematization in a public hospital in Teresina
}

La calidad de la sistematización de la atención de enfermería en un hospital público de

Teresina

Leila Mariane Machado Torres Bezerra1, Lorena Gomes de Abreu Lima1', Nájila Aguiar Freitas Lemos $^{1}$, Francisca Maria Pereira da Cruz ${ }^{1}$, Sara Ferreira Lobato de Brito ${ }^{1}$, Guilherme Pertinni de Morais Gouveia ${ }^{2}$, Gabriela Dantas Carvalho ${ }^{1 *}$, Rayssa Maria de Araujo Carvalho', Renata Fortes Santiago $^{3}$, Roberta Fortes Santiago'.

\section{RESUMO}

Objetivo: Analisar a aplicabilidade da SAE em um hospital de grande porte de Teresina. Métodos: Trata-se de uma pesquisa exploratória, quantitativo, realizado em um hospital público de Teresina-PI. Foram aplicados questionários estruturados aos enfermeiros, seguido da análise dos prontuários, sob aprovação do Comitê de Etica em Pesquisa com Seres Humanos do Centro Universitário UNIFACID (2.271.299). Todos os participantes assinaram o Termo de Consentimento Livre e Esclarecido (TCLE) e para a análise dos prontuários foi assinado o Termo de Consentimento do Uso dos Dados (TCUD). Os dados analisados pelo programa SPSS, e empregado o teste de tendência linear. Foram considerados significantes valores de $\mathrm{p}<0,05$. Resultados: Houve predominância de profissionais do gênero feminino $(76,7 \%, \mathrm{n}=23)$, especializadas $(80 \%, n=24)$, com menos de 5 anos de experiência profissional $(36,7 \%$, $n=11)$. Estes profissionais mostraram ter conhecimento sobre a SAE $(73 \%, n=22)$, no entanto, quando analisados 0 preenchimento das mesmas nos prontuários das clínicas médica, ortopédica e cirúrgica obtiveram avaliação ruim, mostrando não preencherem completamente $(63,3 \%, n=19)$ com ênfase nas clínicas ortopédica e cirúrgica, respectivamente. Conclusão: Constatou-se que a SAE ainda se encontra em fase de implantação no hospital pesquisado e ocorre de forma bastante fragmentada em todos setores investigados.

Palavras-chave: Cuidados de enfermagem, Assistência de enfermagem, Assistência hospitalar, Processos de enfermagem.

\begin{abstract}
Objective: To analyze the applicability of SAE in a large hospital in Teresina. Methods: This is an exploratory, quantitative research, carried out in a public hospital in Teresina-PI. Structured questionnaires were applied to nurses, followed by the analysis of medical records, under the approval of the Ethics Committee for Research with Human Beings at the UNIFACID University Center $(2,271,299)$. All participants signed the Free and Informed Consent Form (ICF) and for the analysis of the medical records, the Data Use Consent Form (TCUD) was signed. The data analyzed by the SPSS program, and the linear trend test was used. Values of $p<0.05$ were considered significant. Results: There was a predominance of female professionals $(76.7 \%, n=23)$, specialized $(80 \%, n=24)$, with less than 5 years of professional experience $(36.7 \%, n=11)$. These professionals showed to have knowledge about SAE $(73 \%, n=22)$, however, when analyzing their filling in the medical, orthopedic and surgical medical records, they obtained a bad evaluation, showing that they did not completely fill it out $(63.3 \%, n=19)$ with an emphasis on orthopedic and surgical clinics, respectively. Conclusion: It was found that SAE is still in the implementation phase in the researched hospital and occurs in a very fragmented way in all investigated sectors.
\end{abstract}

Key words: Nursing care, Nursing assistance, Hospital assistance, Nursing processes.

${ }^{1}$ Centro Universitário UNIFACID, Teresina - PI. *E-mail: ftgabrieladantas@hotmail.com

2 Universidade do Delta do Parnaíba, Parnaíba - PI.

${ }^{3}$ Faculdade UNIRB, Parnaíba - PI. 


\section{RESUMEN}

Objetivo: Analizar la aplicabilidad de SAE en un gran hospital de Teresina. Métodos: Se trata de una investigación exploratoria, cuantitativa, realizada en un hospital público de Teresina-PI. Se aplicaron cuestionarios estructurados a enfermeras, seguidos del análisis de historias clínicas, bajo la aprobación del Comité de Ética para la Investigación con Seres Humanos del Centro Universitario UNIFACID (2.271.299). Todos los participantes firmaron el Formulario de Consentimiento Libre e Informado (ICF) y para el análisis de la historia clínica se firmó el Formulario de Consentimiento de Uso de Datos (TCUD). Se utilizaron los datos analizados por el programa SPSS y la prueba de tendencia lineal. Los valores de $p<0,05$ se consideraron significativos. Resultados: Hubo predominio de mujeres profesionales $(76,7 \%, n=23)$, especializadas $(80 \%$, $n=24)$, con menos de 5 años de experiencia profesional $(36,7 \%, n=11)$. Estos profesionales demostraron tener conocimientos sobre SAE (73\%, $\mathrm{n}=22)$, sin embargo, al analizar su cumplimentación de la historia clínica médica, ortopédica y quirúrgica, obtuvieron una mala valoración, demostrando que no cumplimentaron completamente $(63,3 \%), n=19)$ con énfasis en clínicas ortopédicas y quirúrgicas, respectivamente. Conclusión: Se encontró que el SAE aún se encuentra en fase de implementación en el hospital investigado y se presenta de manera muy fragmentada en todos los sectores investigados.

Palabras clave: Atención de enfermería, Asistencia de enfermería, Asistencia hospitalaria, Procesos de enfermería.

\section{INTRODUÇÃO}

A Sistematização da Assistência da Enfermagem (SAE) é uma atividade que orienta a prática da equipe de enfermagem, com a participação efetiva e direta dos enfermeiros, técnicos e auxiliares de enfermagem. De acordo com o Conselho Federal de Enfermagem (COFEN), é uma metodologia que consiste na organização, sistematização e execução das ações congruentes, realizadas pela equipe durante o período em que o cliente é assistido pelos serviços de saúde, de modo a direcionar um cuidado integral e individualizado, possibilitando, desta forma, uma melhoria na qualidade da assistência (COFEN, 2010).

Em 2002, por meio da resolução do COFEN no 272/2002, tornou-se obrigatória a implantação e implementação da SAE em todas as instituições de saúde pública e privada (MANGUEIRA SO, et al., 2012). Essa resolução foi revogada após sete anos de aplicabilidade, sendo substituída pela resolução COFEN $\mathrm{n}^{\circ}$ $358 / 2009$, a qual determina a obrigatoriedade e a necessidade de sua aplicação na prática cotidiana da enfermagem, além de preconizar como atividade privativa do enfermeiro, baseada em estratégias científicas planejadas para a identificação das diversas situações do processo saúde/doença (NERY SI, et al., 2013).

A SAE é de grande relevância para os profissionais da enfermagem, uma vez que funciona como instrumento de autonomia e desenvolvimento para uma prática voltada para o conhecimento científico. Estudos mostram que grande parte dos profissionais demonstram interesse quanto à sistematização (TORRES E, et al., 2011 e BARRETO MS, et al., 2020), no entanto, percebe-se que, apesar da sua importância, alguns profissionais ainda resistem à sua aplicação, evidenciando-a como uma burocratização da assistência, um processo difícil e complexo, algo que demanda tempo, falta de domínio, entre outros (CASTRO RR, et al., 2016). Diante disso, observa-se que a sistematização não se encontra totalmente implantada nos serviços de saúde, como também muitas dificuldades são encontradas na implementação desse processo (DUTRA LP, 2014).

De acordo com a Resolução 359/2009, a SAE é constituída de cinco etapas, compreendendo o histórico de enfermagem, que consiste na coleta dos dados e do exame físico; diagnóstico da enfermagem, processo deliberado, sistemático e contínuo, realizado com o auxílio de métodos e técnicas variadas que têm por finalidade a obtenção de informações; planejamento da enfermagem, no qual se elabora um plano assistencial com metas a serem alcançadas; implementação de enfermagem, momento em que se põe em prática as ações determinadas no planejamento; e por fim, a avaliação da enfermagem, processo deliberado, sistemático e contínuo de verificação de mudanças para determinar se as ações ou intervenções alcançaram o resultado esperado, e de verificação da necessidade de mudanças ou adaptações nas etapas do processo de enfermagem (GOUVEIA HG, 2004; BRASIL, 2009). 
A SAE é essencial no cuidado da saúde, uma vez que essa ferramenta contempla o paciente na sua recuperação, a partir do conhecimento específico, de uma reflexão crítica e de questionamentos acerca da organização e filosofia do trabalho de enfermagem. Integrar a SAE é dotar a enfermagem de cientificidade, promovendo o cuidado. É importante que o profissional da enfermagem, responsável pela equipe e pelo cuidado com o paciente, tenha conhecimento, habilidade e competência sobre o modelo de saúde que será executado para gerenciar a assistência de modo seguro e organizado (MARINELLI NP, et al., 2015).

A aplicabilidade da SAE nos serviços de saúde funciona abaixo do esperado, isso ocorre na maioria das instituições, particularmente, por falta de conhecimento e de como aplicar corretamente os questionários usados para a implantação, bem como pela sobrecarga de trabalho, expressa pelo número insuficiente de profissionais qualificados e aptos a identificar os problemas reais e potenciais dos pacientes envolvidos no processo de enfermagem (MARINELLI NP, et al., 2015).

Dada a importância da SAE como um reconhecimento profissional científico, valorizando a categoria profissional de enfermeiros, além de garantir a prestação de cuidados ao paciente de forma holística, organizada e com embasamento científico, proporcionando à enfermagem o poder de decisão, o estudo busca analisar a aplicabilidade da SAE em um hospital de grande porte de Teresina, visando sensibilizar os profissionais de enfermagem quanto à prática adequada da SAE e sua avaliação periódica por parte do enfermeiro que gerencia a equipe de enfermagem para que se torne viável na realidade do serviço.

\section{MÉTODOS}

Trata-se de uma pesquisa descritiva, exploratória, prospectiva e transversal, de caráter quantitativo. Teve como cenário do estudo um hospital público de grande porte localizado no município de Teresina-PI, onde foram avaliadas a implementação da SAE nos setores das clínicas hospitalares médica, cirúrgica e ortopédica, no período de janeiro a agosto de 2019. A escolha dessas clínicas se deu por assistirem, especificamente, pacientes adultos e por terem alta rotatividade. A população do estudo foi composta pelos profissionais enfermeiros que fazem parte dessas clínicas, por um tempo mínimo de 6 meses, que tem conhecimento do SAE e que aceitaram participar do estudo.

Foram utilizados para coleta de dados dois questionários estruturados, elaborados pelos autores para 0 respectivo trabalho, contemplando 6 e 15 questões, respectivamente. Um questionário foi aplicado aos profissionais de enfermagem, onde foi abordada a utilização da SAE e as dificuldades e benefícios da sua implantação no processo de trabalho do enfermeiro. O segundo questionário foi empregado para avaliação da SAE registrada nos prontuários. Com base nos dados preenchidos nos prontuários, foi classificado como: boa, quando todas as etapas eram realizadas completamente; regular quando todas as etapas eram realizadas, porém de forma incompleta; e ruim quando não eram realizadas.

A presente pesquisa foi realizada de acordo com as Diretrizes para pesquisa envolvendo humanos preconizadas pela Resolução do Conselho Nacional de Saúde 466/12, sendo a mesma submetida à apreciação do Comitê de Ética em Pesquisa com Seres Humanos do Centro Universitário UNIFACID e aprovada sem restrições sob o número de parecer 2.271.299. Todos os participantes assinaram o Termo de Consentimento Livre e Esclarecido (TCLE) e para a análise dos prontuários, o trabalho seguiu com a aprovação do Comitê de Ética do referido hospital, sendo assinado o Termo de Consentimento do Uso dos Dados (TCUD).

Os dados foram organizados em planilha Excel, seguido da análise estatística pelo programa Statitical Package for the Social Science (SPSS), sendo empregado o teste de tendência linear. Foram considerados significantes valores de $p<0,05$. Os dados foram expressos em tabelas.

\section{RESULTADOS}

Os dados coletados foram divididos em duas sessões: a avaliação da aplicabilidade da SAE pela percepção dos profissionais da enfermagem e a aplicabilidade da SAE nos prontuários. 


\section{Avaliação da aplicabilidade da SAE pela visão dos enfermeiros}

Participaram da pesquisa 30 enfermeiros, em sua maioria, do gênero feminino (76,7\%, $n=23)$, formados entre 5 e 10 anos $(50 \%, n=15)$, prevalecendo profissionais especialistas $(80 \%, n=24)$, com tempo de atuação profissional menor que 5 anos $(36,7 \%, n=11)$ e entre 5 e 10 anos $(30 \%, n=9)$, respectivamente (Tabela 1$)$.

Tabela 1 - Perfil dos enfermeiros participantes do estudo $(n=30)$. Teresina, PI, 2019.

\begin{tabular}{llll}
\hline Perfil dos enfermeiros & & $\mathbf{n}$ & $\%$ \\
\hline \multirow{2}{*}{ Gênero } & Feminino & 23 & 76,7 \\
& Masculino & 7 & 23,3 \\
\hline \multirow{4}{*}{ Tempo de conclusão } & Menos de 5 anos & 5 & 16,7 \\
& Entre 5 e 10 anos & 15 & 50,0 \\
& Entre 10 e 15 anos & 7 & 23,3 \\
& Entre 15 e 20 anos & 2 & 6,7 \\
& Mais de 20 anos & 1 & 3,3 \\
\hline \multirow{3}{*}{ Título da pós-graduação } & Especialista & 24 & 80,0 \\
& Mestre & 2 & 6,7 \\
& Nenhum & 3 & 10,0 \\
& Especialista e mestre & 1 & 3,3 \\
\hline \multirow{3}{*}{ Tempo de trabalho } & Menos de 5 anos & 11 & 36,7 \\
& Entre 5 e 10 anos & 9 & 30,0 \\
& Entre 10 e 15 anos & 7 & 23,3 \\
& Entre 15 e 20 anos & 2 & 6,7 \\
& Mais de 20 anos & 1 & 3,3 \\
\hline
\end{tabular}

Legenda: $\mathrm{n}=$ Número de participantes; \%= percentual. Fonte: Bezerra LMMT, et al., 2021.

No que se refere à aplicação da SAE, na Tabela 2, apenas uma pequena parcela dos enfermeiros informou realizá-la em sua totalidade $(26,7 \%, n=8)$, porém, quando analisado o conhecimento dos profissionais quanto a sequência correta para a realização das etapas da SAE prevaleceu aqueles que afirmaram ter conhecimento sobre seu procedimento $(73,3 \%, \mathrm{n}=22)$.

Tabela 2 - Distribuição das características relacionadas à SAE e sua aplicabilidade $(n=30)$. Teresina, PI, 2019.

\begin{tabular}{llccc}
\hline Características da SAE e sua aplicabilidade & $\mathbf{n}$ & $\%$ \\
\hline \multirow{2}{*}{ Aplica SAE } & Totalmente & Parcialmente & 8 & 26,7 \\
& Não aplico & 19 & 63,3 \\
& Avaliação, Histórico, Diagnóstico, Prescrição de enfermagem em & 3 & 10,0 \\
\hline \multirow{3}{*}{$\begin{array}{l}\text { Sequência } \\
\text { correta }\end{array}$} & Implementação de enfermagem & 5 & 16,7 \\
& Histórico, Avaliação, Diagnóstico e Prescrição de enfermagem & 3 & 10,0 \\
& Histórico, Diagnóstico, Prescrição de enfermagem, Implementação e e & 22 & 73,3 \\
& Avaliação & & \\
\hline
\end{tabular}

Legenda: $\mathrm{n}=$ Número de participantes; \%= percentual; Fonte: Bezerra LMMT, et al., 2021.

Os enfermeiros participantes do estudo descreveram suas opiniões relacionadas à importância, benefícios, dificuldades e fatores desmotivadores relacionados à aplicação da SAE. A maioria (33,3\%) destacou que a 
importância da SAE para a prática da enfermagem está relacionada a todos esses aspectos: organização e padronização da assistência, individualização do cuidado, continuidade da assistência e melhoria para instituição. Em relação ao grau de importância da SAE para a assistência de enfermagem, todos (100\%) a classificaram como muito importante; para a maioria $(50 \%)$, os benefícios da SAE para a prática de enfermagem consistem simultaneamente na assistência ao cliente precisa e organizada, autonomia para tomada de decisões e assistência humanizada.

Predominou entre fatores que dificultam a aplicação da SAE o número reduzido do quadro de profissionais $(43,3 \%)$ e a sobrecarga de trabalho $(43,3 \%)$. Já entre os fatores que desmotivam sua aplicação, a alta demanda de pacientes $(36,7 \%)$ e a falta de interesse por parte da instituição $(36,7 \%)$.

\section{Avaliação da aplicabilidade da SAE nos prontuários}

Foram analisados 379 prontuários. Os dados apresentados na Tabela 3 descrevem o preenchimento do SAE em 4 fases, considerando o histórico, diagnóstico, prescrição e avaliação da enfermagem, onde podese observar que a maior parte do histórico $(68,6 \%, n=260)$, do diagnóstico $(98,1 \%, n=372)$, da prescrição $(99,7 \%, n=378)$ e da avaliação $(94,7 \%, n=359)$ da enfermagem foi avaliada como ruim ou não realizada.

Tabela 3 - Associação entre a qualidade de preenchimento do histórico de enfermagem com o setor de preenchimento $(n=379)$. Teresina, PI, 2019.

\begin{tabular}{|c|c|c|c|c|}
\hline \multirow{2}{*}{ Setor } & \multicolumn{3}{|c|}{ Preenchimento do histórico de enfermagem } & \multirow{2}{*}{ Valor de $p$} \\
\hline & Boa & Regular & Ruim & \\
\hline Ortopédico & $0(0,0 \%)$ & $2(1,8 \%)$ & $239(91,9 \%)$ & \multirow{4}{*}{$<0,001$} \\
\hline Cirúrgico & $6(66,7 \%)$ & $93(84,5 \%)$ & $20(7,7 \%)$ & \\
\hline Médico & $3(33,3 \%)$ & $15(13,6 \%)$ & $1(0,4 \%)$ & \\
\hline Total & $9(100 \%)$ & $110(100 \%)$ & $260(100 \%)$ & \\
\hline \multirow{2}{*}{ Setor } & \multicolumn{3}{|c|}{ Preenchimento do diagnóstico de enfermagem } & \multirow{2}{*}{ Valor de $p$} \\
\hline & Boa & Regular & Ruim & \\
\hline Ortopédico & $0(0,0 \%)$ & $0(0,0 \%)$ & $241(64,8 \%)$ & \\
\hline Cirúrgico & $0(0,0 \%)$ & $0(0,0 \%)$ & $119(32 \%)$ & \\
\hline Médico & $2(100 \%)$ & $5(100 \%)$ & $12(3,2 \%)$ & $<0,001$ \\
\hline Total & $2(100 \%)$ & $5(100 \%)$ & $372(100 \%)$ & \\
\hline \multirow[b]{2}{*}{ Setor } & \multicolumn{3}{|c|}{ Preenchimento da prescrição de enfermagem } & \multirow{2}{*}{ Valor de $p$} \\
\hline & Boa & Regular & Ruim & \\
\hline Ortopédico & $0(0,0 \%)$ & - & $241(63,8 \%)$ & \\
\hline Cirúrgico & $0(0,0 \%)$ & - & $119(31,5 \%)$ & \\
\hline Médico & $1(100 \%)$ & - & $18(4,8 \%)$ & 0,050 \\
\hline Total & $1(100 \%)$ & - & $378(100 \%)$ & \\
\hline \multirow{2}{*}{ Setor } & \multicolumn{3}{|c|}{ Preenchimento da avaliação de enfermagem } & \multirow{2}{*}{ Valor de $p$} \\
\hline & Boa & Regular & Ruim & \\
\hline Ortopédico & $0(0,0 \%)$ & $1(100 \%)$ & $241(67,1 \%)$ & \multirow{4}{*}{$<0,001$} \\
\hline Cirúrgico & $0(0,0 \%)$ & $3(18,8 \%)$ & $116(32,3 \%)$ & \\
\hline Médico & $4(100 \%)$ & $13(81,2 \%)$ & $2(0,6 \%)$ & \\
\hline Total & $4(100 \%)$ & $16(100 \%)$ & $359(100 \%)$ & \\
\hline
\end{tabular}

Legenda: ${ }^{*} p$ valor estatisticamente significante (Teste de tendência linear).

Fonte: Bezerra LMMT, et al., 2021.

No que se refere à primeira etapa, o histórico da enfermagem, verificou-se que, apenas $2,3 \%(n=9)$ foi considerado de boa qualidade, $29 \%(n=110)$ como regular e $68,6 \%(n=260)$ como ruim, tendo como o setor clínico da ortopedia como o mais crítico com $0 \%(n=0)$ com bom preenchimento e $91,9 \%(n=239)$ avaliado como preenchimento ruim ou não realizado.

$\mathrm{Na}$ etapa seguinte, referente ao diagnóstico da enfermagem, somente $0,5 \%(n=2)$ foi apontado como de boa qualidade e $98,1 \%$ ( $n=372)$ como ruim. Semelhante ao histórico da enfermagem, o diagnóstico da clínica ortopédica se mantém como o mais crítico $(63,8 \%, n=241)$, seguido da clínica cirúrgica $(32 \%, n=119)$. 
A terceira etapa, que consiste na prescrição da enfermagem, não houve associação entre a qualidade de preenchimento da prescrição de enfermagem com os setores avaliados. Esta etapa foi a mais crítica, apresentando o maior percentual de insatisfação quanto a atuação da enfermagem na SAE com um total de $99,7 \%$ ( $n=378)$ avaliações ruins ou não realizadas, mantendo-se a clínica ortopédica $(63,5 \%, n=241)$ como a pior avaliada, seguida da clínica cirúrgica $(31,4 \%, n=119)$.

Na quarta etapa, avaliação da enfermagem, apenas $1 \%(n=4)$ foi qualificada em boa qualidade, enquanto que, $94,7 \%(n=359)$ destacaram-se como ruim. Semelhante as etapas anteriores, destacam-se as clínicas ortopédica $(67,1 \%, n=241)$ e a cirúrgica $(32,3 \%, n=116)$ como as piores avaliadas.

A implementação de enfermagem é apresentada na Tabela 4, observando que a implementação não foi realizada pelos setores, conferindo que a aplicabilidade prática da SAE não foi satisfatória na instituição avaliada, o que implica no menor conhecimentos técnicos e científicos da equipe para organizar, planejar, executar ações e instrumentalizar a equipe responsável pela assistência.

Tabela 4: Associação entre a qualidade de preenchimento da implementação de enfermagem com o setor de preenchimento $(\mathrm{n}=379)$. Teresina, PI, 2019.

\begin{tabular}{lc}
\hline \multirow{2}{*}{ Setor } & Qualidade de preenchimento da evolução da enfermagem \\
\cline { 2 - 2 } & Ruim $(\mathrm{n} ; \%)$ \\
\hline Ortopédico & $241 ;(63,6 \%)$ \\
Cirúrgico & $119 ;(31,4 \%)$ \\
Médico & $19 ;(5,0 \%)$ \\
\hline Total & $379 ;(100,0 \%)$ \\
\hline Legenda: $n=$ Número de participantes: \%= percentual; Fonte: Bezerra LMMT, et al. 2021.
\end{tabular}

Legenda: $\mathrm{n}=$ Número de participantes; \%= percentual; Fonte: Bezerra LMMT, et al., 2021.

\section{DISCUSSÃO}

O enfermeiro vivencia um desafio na construção e seleção do conhecimento sobre o qual se respalda seu empenho gerencial e assistencial. O desenvolvimento do processo de trabalho da enfermagem, para efetivar a proposta de viabilizar, mover, manter ou recuperar o nível de saúde do paciente se faz presente. Assim, a SAE vem para somar e configurar o planejamento, a execução, o controle e a avaliação das ações de cuidados aos pacientes (GONÇALVES LRR, et al., 2007).

A evolução da enfermagem atrela-se às mudanças vivenciadas na sociedade, levando os seus profissionais a se questionarem e a refletirem sobre a sua situação prática (MARINELLI NP, 2015). Partindo desta necessidade de adequação surge o SAE, um sistema de organização reconhecido internacionalmente que visa direcionar o trabalho profissional, tendo como objetivo principal sistematizar e qualificar 0 atendimento ao paciente, família e comunidade (OLIVEIRA MR, et al., 2019).

Segundo Barreto MS, et al. (2020) a utilização deste instrumento garante ao enfermeiro identificar as necessidades de cada paciente/grupo, direcionando o atendimento a partir de prioridades estabelecidas. No entanto, apesar da integração da SAE ser obrigatória, sua realização, muitas vezes, não é cumprida e/ou empreendida de forma correta ou completa. Egilegor HJX, et al. (2018) descrevem que essa realidade não se limita as demandas do serviço público brasileiro. Segundo os autores, na Espanha, comparada aos serviços prestados nos Estados Unidos e Canadá, apresenta fragilidades na implementação da SAE, semelhantes as dificuldades encontradas no Brasil.

Partindo dos inúmeros estudos encontrados que pontuam a dificuldade da implantação do SAE nos serviços da enfermagem brasileira, bem como a sua realização na prática clínica, buscou-se investigar se a realidade dos profissionais da enfermagem atuantes em um grande hospital público de Teresina, se assemelha ao perfil apresentado.

Segundo Machado ME, et al. (2016), a área da saúde abrange um contingente de 3,5 milhões de trabalhadores, no qual $50 \%$ são representados pela enfermagem. Refere-se a uma categoria fortemente inserida no Sistema Único de Saúde e com atuação nos setores público, privado, filantrópico e de ensino. É 
uma classe, hegemonicamente, feminina, representada por $84,6 \%$ das mulheres. De acordo com o presente trabalho, observou-se uma predominância do gênero feminino, em sua maioria, especializadas, com tempo médio de 5 anos de atuação no mercado de trabalho. Resultado semelhante foi encontrado por Da Silva FML, et al. (2019) ao analisarem as dificuldades enfrentadas por enfermeiros atuantes na Unidade de Terapia Intensiva adulta, destacando como as principais dificuldades encontradas pelos profissionais na implementação do SAE a sobrecarga de trabalho e o conhecimento superficial da equipe sobre o SAE.

Segundo Castro RR, et al. (2016), na prática, a aplicabilidade da SAE nos serviços de saúde funciona abaixo do esperado, isso ocorre na maioria das instituições, independente do caráter público ou privado, sobretudo, por falta de conhecimento e de como aplicar corretamente os questionários usados para a implantação, bem como pela sobrecarga de trabalho, evidenciada pelo número insuficiente de profissionais qualificados e capazes de identificar os problemas reais e potenciais dos pacientes.

Observa-se que nos resultados apresentados, há uma baixa incidência dos profissionais que admitem realizar o SAE completamente, isso é comprovado por meio da análise dos prontuários, onde, em sua maioria, não encontram-se com as informações completas, de tal modo que, para todas as etapas apresentadas, todas foram classificadas como ruim e/ou não realizadas, com maior destaque para as clínicas ortopédicas e cirúrgica, acredita-se que seja decorrente da demanda do setor, contudo, inúmeros estudos pontuam a dificuldade de associar aquilo que é aprendido no campo teórico e sua aplicação no campo prático, bem como a não valorização das suas atividades, sendo o principal obstáculo identificado a alta demanda de atividades impostas a esses profissionais, de modo que não conseguem em sua completude desempenhar todas as atividades demandadas. Independente do motivo que tenha levado à desmotivação dos enfermeiros, o descrédito referente à utilização, enquanto ferramenta qualificadora da assistência e promotora da autonomia profissional, leva a não adesão à sua aplicabilidade.

Desde o início da implantação da SAE, foram constatadas as possíveis dificuldades a serem encontradas, com destaque para a complexidade e falta de uniformidade no estabelecimento de suas etapas, insegurança dos profissionais para realizar as atividades inerentes ao processo, dado que é um processo novo e que requer preparo prévio e número de enfermeiros insuficiente para desempenhar todas as obrigatoriedades exigidas. Acredita-se que estes fatores influenciam para que muitos enfermeiros deixem de sistematizar sua assistência, fragmentando o cuidado realizado (MEDEIROS AL, et al., 2013).

Partindo da observação feita por Medeiros AL, et al. (2013), vale ressaltar que ao analisarem os prontuários buscou-se classifica-los quanto à qualidade do seu preenchimento que, no geral, não aconteceu, ou aconteceu de forma incompleta, sem constar a assinatura e/ou sem o carimbo do profissional enfermeiro responsável. Dentro deste contexto, uma pesquisa realizada por Castro RR, et al. (2016) ao analisar as compreensões e desafios acerca da sistematização da assistência de enfermagem no setor da clínica médica de um hospital público do município de Mossoró (Rio Grande do Norte), segundo a percepção da equipe de enfermagem, para o enfermeiro desenvolver suas atribuições não é preciso a aplicação de todo o processo de enfermagem, mas de pelo menos parte dele, mostrando que além das dificuldades encontradas pelo próprio sistema de trabalho, parte dos profissionais consideram que a SAE é desnecessária para que a assistência de qualidade seja prestada.

Embora a SAE ofereça ao enfermeiro a viabilidade de organizar seu trabalho baseado em uma filosofia e uma metodologia que elege um cuidado individualizado, excelência na qualidade, com consequente visibilidade e credibilidade do seu trabalho, eles ainda preferem manter o antigo modelo assistencial, como mostra uma pesquisa realizada em um hospital público, na cidade de Recife, onde os enfermeiros executam apenas duas etapas: exame físico e a avaliação de enfermagem, descontextualizadas dos verdadeiros princípios e da utilidade da SAE para o avanço da qualidade da assistência ao paciente (SOUZA, et al., 2015).

Empregar a SAE de forma segmentada pode interferir no estímulo do profissional enfermeiro e comprometer a integração das etapas. Efetuar ações não sistematizadas pode prejudicar o planejamento do cuidado prestado ao paciente, ao dificultar a avaliação deste cuidado (OLIVEIRA MR, et al., 2012).

O enfermeiro tem o papel de envolver toda a equipe de enfermagem, quanto à importância da sistematização do cuidado, para o comprometimento e participação ativa de todos. Entretanto, mesmo com 
as dificuldades relacionadas à implementação da SAE, verifica-se o reconhecimento dos enfermeiros quanto à necessidade de sistematizar o cuidado, colaborando como um fator incentivador e uma preocupação em doar-se à execução das atividades garantindo a assistência, e os benefícios que a sistematização pode disponibilizar ao cliente e ao enfermeiro (GOMES LA, 2012).

É importante destacar que o dimensionamento de pessoal, quando realizado de acordo com as necessidades da instituição, proporciona ao profissional maior comodidade para organizar, executar e avaliar os planos de cuidado, considerando um fator determinante para que a SAE seja implementada (MAYA CM, 2011).

Encontrou-se como limitação deste estudo um quantitativo muito alto de prontuários, o que demandou uma atenção redobrada de tempo para dispor da análise que contradiz ao tempo disponível para obtenção dos dados pelos profissionais atuantes, que por apresentarem um número reduzido, com uma alta demanda de atividades, dificultou que a entrevista pudesse ser aprofundada, visando compreender melhor a sua percepção sobre a SAE, suas limitações e dificuldades de implantação, o que sugere-se que estudos futuros possam abordar melhor o papel da enfermagem, bem como da gestão hospitalar como instrumentos de melhorias para a execução da SAE, uma vez que este estudo já possibilitou a configuração da realidade apresentada. Além disso, considerando que a pesquisa foi restrita a um único hospital público de Teresina, acredita-se que para melhor configurar o perfil da implantação da SAE local, seja necessário investigar outras instituições como modo de melhor delinear a realidade da SAE nos serviços públicos de Teresina.

\section{CONCLUSÃO}

Constatou-se que a SAE ainda se encontra em fase de implantação no hospital pesquisado e ocorre de forma bastante fragmentada nos setores investigados. Os enfermeiros mostram ter conhecimento sobre 0 que é a ferramenta da SAE e suas etapas, contudo, a percepção destes ainda não se faz clara a respeito da sua aplicabilidade. É observado em suas falas a percepção da importância da SAE para o desenvolvimento do trabalho da enfermagem, no entanto, esta mostra divergência quanto a sua prática clínica, onde muitos ainda preferem o modo tradicional de notificação e norteamento dos serviços desenvolvidos. Diante do exposto, vê-se a necessidade de uma reorganização na assistência prestada pelos enfermeiros dos setores avaliados do hospital de estudo, propondo-se a educação permanente como estratégia de investimento e conscientização, para que haja o envolvimento destes profissionais, bem como da instituição neste processo, visando a melhoria da qualidade da assistência de enfermagem aos pacientes.

\section{REFERÊNCIAS}

1. BARRETO MS, et al. Sistematização da assistência de enfermagem: a práxis do enfermeiro de hospital de pequeno porte. Escola Anna Nery,2020; 24(4): 1-8.

2. BRASIL. Conselho Federal de Enfermagem Resolução COFEN-358/2009. Disponível em: http://www.cofen.gov.br/resoluo-cofen-3582009_4384.html. Acesso em 08 de nov. de 2018

3. BRASIL. Conselho Federal de Enfermagem. CŌREN - RO Realiza o curso de Gerenciamento e Sistematização da Assistência de Enfermagem - SAE, 2010. Disponível em:<http://www.cofen.gov.br/coren-ro-realiza-o-curso-degerenciamento-e-sistematizaao-da-assistencia-de-enfermagem-sae_5478.html>. Acesso em 30 mar. 2017

4. BRASIL. Conselho Federal de Enfermagem.Cofen lança Perfil da Enfermagem no Piauí. 2015. Disponível em: http://www.cofen.gov.br/cofen-lanca-perfil-da-enfermagem-no-piaui_32930.html. Acesso em 14 nov. de 2018.

5. CAMELO SHH. Políticas De Recursos Humanos: Sistema Único De Saúde, Bases Legais E Implicações Para A Enfermagem.Rev. enferm. UERJ, 2014; 17(4): 589-94.

6. CASTRO RR, et al. Compreensões e desafios acerca da sistematização da assistência de enfermagem. Rev enferm UERJ, 2016.

7. DA SILVA FML, et al. Dificuldades na implementação da Sistematização da Assistência de Enfermagem na Unidade de Terapia Intensiva Adulto. Revista Eletrônica Acervo Saúde, 2019; 28 : e986.

8. DUTRA LP. Avaliação da Implementação da Sistematização da Asistencia de Enfermagem, uma Revisão Integrativa. Monografia orientada pela Profa. Ma. Kamilla Cristiane de Oliveira Silva (graduação em enfermagem) - Faculdade Integral Diferencial, 2014; 71p.

9. EGILEGOR HJX, et al. The use of the nursing process in Spain as compared to the United States and Canada. Int J Nurs Knowl, 2018;29(3):171-75. 
10. GOMES LA, BRITO DS. Desafios Na Implantação da Sistematização da Assistência de Enfermagem: Uma Revisão De Literatura. Revista Interdisciplinar UNINOVAFAPI, 2012; 5(3):64-70.

11. GONÇALVES LRR, et al. O Desafio De Implantar a Sistematização da Assistência de Enfermagem Sob A Ótica Do Discente. Esc Enferm Anna Nery,2007; 11(3): 459-65.

12. GOUVEIA HG, LOPES MHBM. Diagnósticos de enfermagem e problemas colaborativos mais comuns na gestação de risco. Rev Latino-Am Enfermagem. 2004; 12(2): 175-82.

13. MACHADO ME, et al. Mercado de trabalho em enfermagem no âmbito do SUS: uma abordagem a partir da pesquisa Perfil da Enfermagem no Brasil. Divulgação em Saúde para debate, 2016; 56: 52-69.

14. MANGUEIRA, SO. et al. Implantação da Sistematização da Assistência de Enfermagem: Opinião de uma Equipe de Enfermagem Hospitalar. Enfermagem em Foco, 2012; 3(3): 135-138.

15. MARINELLI NP, et al. Sistematização da Assistência de Enfermagem: Desafios para à implantação. Rev. Enfermagem Contemporânea, 2015; 4(2): 254-263.

16. MAYA CM, SIMÕES ALA. Implicações Do Dimensionamento Do Pessoal De Enfermagem No Desempenho Das Competências do Profissional Enfermeiro. RevBrasEnferm, 2011; 64(5).

17. MEDEIROS AL, et al. Sistematização da assistência de enfermagem: dificuldades evidenciadas pela teoria fundamentada nos dados. Rev enferm UERJ. 2013; 21(1): 47-53.

18. NERY SI, et al. Dificuldades para a Implantação Sistematização da Assistência de Enfermagem em Maternidades. Enfermagem em Foco, 2013; 4 (1): 11-14.

19. OLIVEIRA MR, et al. Sistematização da assistência de enfermagem: percepção e conhecimento da enfermagem Brasileira. Rev Bras Enferm, 2019 ;72(6):1625-31.

20. OLIVEIRA MR, et al. Sistematização da Assistência de Enfermagem na Rede Hospitalar de Uberaba-MG, Revista de Enfermagem Referência - III série- Coimbra, 2012; 8: 105-114.

21. SOUZA NR, et al. Sistematização da Assistência de Enfermagem: Dificuldades Referidas por Enfermeiros de um Hospital Universitário. Ver. Enferm UFPE online, 2015; 9(3).

22. TORRES E, et al. Sistematização da assistência de enfermagem como ferramenta da gerência do cuidado: estudo de caso. Esc Anna Nery (impr.), 2011; 15 (4):730-736. 